\title{
Utilização de técnicas estatísticas em duas revistas de fruticultura
}

\author{
Utilization of statistical techniques in two journals of fruticulture
}

\author{
Tatiana Cantuarias-Avilés ${ }^{\mathrm{I}}$ Carlos Tadeu dos Santos Dias ${ }^{\mathrm{II}}$
}

\begin{abstract}
Neste trabalho são descritas as técnicas de análise estatística utilizadas e a acessibilidade estatística em uma amostra dos artigos originais publicados no período 1996-2006 em duas revistas de pesquisa na área de fruticultura: a Revista Brasileira de Fruticultura (RBF) e a revista francesa Fruits. No total foram classificados 986 artigos em 16 categorias de análise estatística, ordenadas em grau ascendente de complexidade. No período analisado, foi constatado um aumento no uso de análises mais sofisticadas ao longo do tempo em ambos as revistas. Os trabalhos publicados pela RBF aplicaram com maior freqüência técnicas estatísticas mais complexas, com maior utilização de delineamentos em blocos aleatorizados, arranjos fatoriais, parcelas subdivididas e modelos hierárquicos, e do teste de Tukey para comparações múltiplas de médias. Nos trabalhos publicados pela revista Fruits, predominou o uso de outros testes paramétricos e do teste de Duncan. O pacote estatístico SAS foi o mais utilizado nos artigos publicados em ambas as revistas. Os leitores da revista $R B F$ precisaram de um nível de conhecimento estatístico mais elevado para ter acesso à maior parte dos artigos publicados no período, em comparação com os leitores da revista francesa.
\end{abstract}

Palavras-chave: técnicas de análise estatística, acessibilidade estatística, revistas de fruticultura.

\section{ABSTRACT}

The statistical techniques and statistical accessibility were analyzed in a sample collected from the original articles published between 1996 and 2006 by two fruticulture journals: the Revista Brasileira de Fruticultura $(R B F)$ and the French journal Fruits. A total of 986 original articles were classified in 16 classes of statistical analyses, previously ordered by increasing degree of complexity. The complexity of statistical techniques used by both journals increased in time. Along the 1996-2006 period, the articles published in the RBF journal utilized more complex statistical techniques, as well as experimental designs in randomized blocks, factorial, split plot and hierarchical arrangements, and the test of Tukey for mean comparisons. In the articles published by the Fruits journal the use of other parametrical tests and the Duncan test was more frequent. In both journals the SAS statistical software was most frequently utilized. Readers of the $R B F$ journal required a higher level of statistical knowledge to understand the techniques utilized in the articles.

Key words: statistical analysis techniques, fruticulture publications, statistical accessibility.

O aumento no emprego de técnicas estatísticas observado nos últimos anos, nas publicações agronômicas do Brasil e de outros países, promovido pelo contínuo desenvolvimento de poderosos recursos computacionais, tem permitido a utilização de metodologias análíticas de maior complexidade. Em conseqüência, os leitores atuais de

'Departamento de Produção Vegetal, Escola Superior de Agricultura “Luiz de Queiroz” (ESALQ), Universidade de São Paulo (USP). Av. Pádua Dias, 11, CP 9, 13418-900, Piracicaba, SP, Brasil. E-mail: tecavile@esalq.usp.br. Autor para correspondência. IIDepartamento de Ciências Exatas, ESALQ, USP, Piracicaba, SP, Brasil. 
revistas agronômicas estão diante de uma ampla variedade de ferramentas estatísticas e o nível de avaliação crítica dos artigos dependerá do grau de familiaridade com os diversos métodos empregados. A caracterização das técnicas de análise estatística utilizadas em revistas científicas permite determinar a freqüência de uso de cada técnica e estimar a acessibilidade do leitor às metodologias empregadas nos artigos. Com esse objetivo, foram analisados os artigos originais publicados no período 1996-2006 por duas revistas especializadas em fruticultura, uma de abrangência nacional, a Revista Brasileira de Fruticultura (RBF), e outra internacional, a revista francesa Fruits. Essa revista é publicada desde 1945 e tem se especializado na divulgação de pesquisas relacionadas à fruticultura em regiões temperadas e tropicais. A revista Fruits é publicada bimestralmente e está indexada nas bases Biosis Previews, Biological Abstracts, CAB International, CNRS/Pascal e CrossRef, e desde 2001 tem uma versão eletrônica (www.edpsciences.org/Fruits). A RBF, criada em 1978 pela Sociedade Brasileira de Fruticultura, publica quadrimestralmente artigos técnicos e comunicações cientificas, tendo se consolidado nos últimos anos como a principal revista científica na área de fruticultura no Brasil. A RBF está indexada nas bases AGRIS e AGROBASE da CAB Internacional e também é divulgada eletronicamente desde 2001 (www.scielo.br).

O objetivo deste trabalho foi descrever a evolução na utilização de técnicas estatísticas nos últimos anos, em duas revistas especializadas na área de fruticultura. Para isso, foram selecionados todos os artigos publicados em dois números aleatoriamente escolhidos cada ano, entre 1996 e 2006, das revistas Fruits e RBF. Um único revisor examinou 147 artigos originais dos 456 artigos publicados entre 1996 e 2006 pela revista Fruits, e 839 artigos originais, de um total de 1222 publicados no mesmo período pela RBF. Em cada artigo, foram analisadas de forma detalhada as seções de Material e Métodos e Resultados, incluindo tabelas e figuras, e outras seções, em busca de informação adicional sobre a análise dos resultados. A classificação das técnicas estatísticas utilizadas nos artigos foi baseada em uma lista com 16 categorias ordenadas em escala crescente de complexidade, adaptada da lista proposta por RIPOLL et al. (1996) para avaliar métodos estatísticos utilizados em revistas de medicina. Para cada artigo, foram anotadas as categorias de cada uma das análises estatísticas descritas. Não foi considerada a utilização repetida da mesma técnica de análise estatística dentro do mesmo artigo. O grau de conhecimento estatístico que um leitor precisa para compreender todas as técnicas de análises empregadas no artigo, ou seja, a acessibilidade estatística, foi estimada a partir do número de artigos em que uma determinada técnica de análise foi a categoria máxima empregada.

No período 1996-2006, as técnicas de análise utilizadas com maior freqüência em ambas as revistas foram a análise de variância, as comparações múltiplas de médias e a estatística descritiva e gráfica (Tabela 1). Na revista Fruits, foram publicados mais artigos sem análise estatística ou somente com estatística descritiva e gráfica, enquanto que a revista $\mathrm{RBF}$ teve maior publicação de artigos que utilizaram técnicas de análise mais complexas, como a regressão múltipla e a análise multivariada. A comparação dos delineamentos experimentais, testes de comparações múltiplas de médias e pacotes estatísticos utilizados em ambas as revistas no período analisado (Tabela 2) indica que a revista Fruits publicou mais artigos que não utilizaram qualquer delineamento experimental, sendo que a maioria dos casos correspondentes a trabalhos de revisão bibliográfica ou de descrição geral de espécies. Em ambas as revistas os delineamentos mais utilizados foram o inteiramente aleatorizado e o delineamento em blocos aleatorizados. Nos artigos da RBF, houve maior utilização de arranjos fatoriais, parcelas subdivididas e modelos hierárquicos e o teste mais utilizado para comparação de médias foi o de Tukey, enquanto que nos trabalhos publicados na revista Fruits predominou o uso de outros testes paramétricos e do teste de Duncan. Também na revista francesa houve maior utilização do teste $t$ Student e de testes não-paramétricos, comparada com a revista brasileira. O pacote estatístico SAS foi o mais utilizado em pouco mais de 30\% dos artigos publicados em cada revista. Na revista Fruits, foram mais utilizados os pacotes estatísticos Statgraphics Plus 4.0, MSTAT-C, Minitab e programas para análises genéticas. Na RBF foram mais utilizados os pacotes SANEST, SISVAR e SPSS. 
Tabela 1 - Classificação dos artigos originais publicados nas revistas Fruits e Revista Brasileira de Fruticultura (RBF) no período 1996-2006, em 16 categorias de análise estatística.

\begin{tabular}{|c|c|c|c|c|}
\hline \multirow[b]{2}{*}{ Categoria e Tipo de Análise } & \multicolumn{2}{|c|}{$\begin{array}{l}\text { Número de artigos que } \\
\text { efetuam a análise }\end{array}$} & \multicolumn{2}{|c|}{$\begin{array}{l}\text { Porcentagem de artigos } \\
\text { que efetuam a análise }\end{array}$} \\
\hline & $\mathrm{RBF}$ & Fruits & RBF & Fruits \\
\hline 0: sem análise estatística. & 47 & 17 & 5,6 & 11,6 \\
\hline $\begin{array}{l}\text { 1: Estatística Descritiva e Gráfica (percentagens, médias, desvio padrão, erro padrão } \\
\text { da média, histogramas). }\end{array}$ & 207 & 54 & 24,7 & 36,7 \\
\hline 2: Teste t Student para uma ou duas amostras, Teste z, Intervalos de Confiança. & 38 & 8 & 4,5 & 5,4 \\
\hline $\begin{array}{l}\text { 3: Tabelas Bivariáveis (Testes Chi-Quadrado, Teste Exato de Fisher, tabelas de } \\
\text { Contingência). }\end{array}$ & 9 & 6 & 1,1 & 4,1 \\
\hline 4: Análise de Custo e Benefício dos tratamentos. & 12 & 4 & 1,4 & 2,7 \\
\hline 5: Correlação e Regressão Linear Simples. & 108 & 17 & 12,9 & 11,6 \\
\hline 6: Análise de Variância, Análise da Covariância e testes F, Herdabilidade. & 613 & 67 & 73,1 & 45,6 \\
\hline 7: Transformação de Variáveis (logarítmica, raiz quadrada, arcosen, etc.). & 127 & 7 & 15,1 & 4,8 \\
\hline $\begin{array}{l}\text { 8: Testes Não-paramétricos (Teste do Sinal, U de Mann-Witney, t de Wilcoxon, } \\
\text { Kruskal Wallis, Friedman). }\end{array}$ & 9 & 3 & 1,1 & 2,0 \\
\hline $\begin{array}{l}\text { 9: Correlação Não-paramétrica (Rho de Spearman, Tau de Kendall, testes de } \\
\text { tendência). }\end{array}$ & 3 & 0 & 0,4 & 0,0 \\
\hline $\begin{array}{l}\text { 10: Regressão Múltipla (Regressão Polinomial, Regressão Passo a Passo, Modelos } \\
\text { log-lineares). }\end{array}$ & 128 & 2 & 15,3 & 1,4 \\
\hline $\begin{array}{l}\text { 11: Testes de Comparações Múltiplas de Médias (Bonferroni, Scheffé, Duncan, } \\
\text { Newman-Keuls, Tukey, LSD, Contrastes Ortogonais, Intervalos de Confiança } \\
\text { Assintóticos). }\end{array}$ & 519 & 57 & 61,9 & 38,8 \\
\hline $\begin{array}{l}\text { 12: Ajuste e Padronização (padronização das taxas de incidência e prevalência, } \\
\text { probabilidades). }\end{array}$ & 0 & 1 & 0,0 & 0,7 \\
\hline $\begin{array}{l}\text { 13: Analises Multivariadas (Modelos Lineares Mistos (Manova), Análises de } \\
\text { Agrupamento (Cluster), Análise Discriminatória, Análise dos Componentes } \\
\text { Principais e de Fatores). }\end{array}$ & 30 & 3 & 3,6 & 2,0 \\
\hline $\begin{array}{l}\text { 14: Análise de Sobrevivência (tabelas de Vida, Regressão da Sobrevivência } \\
\text { (Regressão Logística, Regressão de Cox) e outras análises de sobrevivência (log rank } \\
\text { test)). }\end{array}$ & 1 & 1 & 0,1 & 0,7 \\
\hline $\begin{array}{l}\text { 15: Outras análises não incluídas nas categorias precedentes (Análise de } \\
\text { Sensibilidade, Bioinformática, Modelos de Superfície de Resposta, Modelos Não } \\
\text { Lineares, etc.). }\end{array}$ & 2 & 0 & 0,2 & 0,0 \\
\hline Número Total de Artigos Analisados & 839 & 147 & & \\
\hline
\end{tabular}

Na análise da acessibilidade estatística dos artigos, foram comparadas as técnicas estatísticas utilizadas nos artigos, agrupadas em três classes: i) artigos que não utilizaram técnicas estatísticas na análise dos dados (categoria 0); ii) artigos que utilizaram como máximo técnicas de análise das categorias 1 (estatística descritiva e gráfica) a 5 (correlação e regressão linear simples), e iii) artigos que utilizaram como máximo técnicas de análise iguais ou superiores à categoria 6 (análise da variância) (Tabela 3). No período 1996-2006, a revista Fruits publicou uma maior percentagem de artigos sem análise estatística e de artigos que utilizaram, como máximo, até a correlação e a regressão linear simples, indicando que os artigos dessa revista foram mais accessíveis para leitores com conhecimentos básicos de técnicas de análise estatística. A partir de 2001, na revista Fruits, aumentou a publicação de artigos que utilizaram técnicas estatísticas mais complexas (categorias 6 a 15), enquanto que na RBF o uso desse tipo de técnicas foi maior e mais estável ao longo de todo o período considerado.

A comparação das técnicas estatísticas empregadas nos artigos publicados entre 1996 e 2006 nas revistas Fruits e RBF revelou um crescente uso de técnicas de análise mais complexas, que fizeram aumentar o nível de conhecimento estatístico exigido aos leitores de ambas as revistas para compreender as técnicas utilizadas nas pesquisas. No entanto, os leitores da revista RBF precisaram de um nível de conhecimento estatístico ainda mais elevado para ter acesso à maior parte dos artigos publicados, em comparação com os leitores da revista Fruits. 
Tabela 2 - Percentagem de artigos publicados em duas revistas de fruticultura no período de 1996 a 2006 , classificados segundo o delineamento experimental, os testes de comparação de médias e os pacotes computacionais utilizados para análise estatística.

\begin{tabular}{|c|c|c|}
\hline \multirow{2}{*}{$\frac{\text { Item }}{\text { I. Delineamentos experimentais }}$} & \multicolumn{2}{|c|}{$\%$ do total de artigos publicados } \\
\hline & Revista Fruits & Revista RBF \\
\hline Sem & 41,5 & 7,1 \\
\hline Modelo linear & 2,7 & 16,5 \\
\hline Inteiramente casualizado & 28,6 & 27,0 \\
\hline Blocos aleatorizados & 21,1 & 24,0 \\
\hline Fatorial & 4,1 & 20,0 \\
\hline Parcelas subdivididas & 2,0 & 5,3 \\
\hline Hierárquico & 0,0 & 0,1 \\
\hline II. Testes de Comparações Múltiplas de Médias & Revista Fruits & Revista RBF \\
\hline t Student & 9,0 & 4,9 \\
\hline Duncan & 29,9 & 17,5 \\
\hline Tukey & 17,9 & 62,1 \\
\hline Outros testes paramétricos ${ }^{1}$ & 31,3 & 12,2 \\
\hline Testes não paramétricos ${ }^{2}$ & 11,9 & 3,3 \\
\hline III. Pacotes estatísticos & Revista Fruits & Revista RBF \\
\hline SAS & 30,3 & 30,4 \\
\hline Statgraphics Plus 4.0 & 12,1 & 3,6 \\
\hline SANEST & 3,0 & 15,9 \\
\hline SISVAR & 0,0 & 8,7 \\
\hline MSTAT-C & 12,1 & 1,5 \\
\hline Minitab & 6,1 & 1,5 \\
\hline SPSS & 3,0 & 4,4 \\
\hline Programas para análises genéticas ${ }^{3}$ & 9,1 & 6,5 \\
\hline Outros ${ }^{4^{*}}$ & 24,3 & 27,5 \\
\hline
\end{tabular}

${ }^{1}$ Testes de Newman-Keuls, LSD, Scott-Knott, Dunnett, etc. ${ }^{2}$ Testes do Sinal, U de Mann-Witney, $t$ de Wilcoxon, Kruskal Wallis, Friedmann, etc. ${ }^{3}$ GENES, GENSTAT 5.1, GENEPOP, SAEG, BIOSYS, GENETIX, JOINMAP, NTSYS, etc. ${ }^{4}$ TABLE CURVE, STATISTICA; STATPAK, COHORT, STAT BOX PRO, STATITCF, etc.

Tabela 3 - Percentagem do total de artigos publicados entre 1996 e 2006 nas revistas Fruits e RBF que utilizaram, como máximo, as distintas categorias de análise estatística.

\begin{tabular}{|c|c|c|c|c|c|c|}
\hline \multirow{2}{*}{ Ano } & \multicolumn{3}{|c|}{--------------------------Revista Fruits------------------------- } & \multicolumn{3}{|c|}{--------------------------'Revista RBF-------------------------- } \\
\hline & Categoria 0 & Categorias 1 a 5 & Categorias 6 a 15 & Categoria 0 & Categorias 1 a 5 & Categorias 6 a 15 \\
\hline 1996 & 0,0 & 64,3 & 35,7 & 8,8 & 29,4 & 61,8 \\
\hline 1997 & 0,0 & 91,7 & 8,3 & 13,9 & 2,8 & 83,3 \\
\hline 1998 & 21,4 & 64,3 & 14,3 & 12,8 & 12,8 & 74,4 \\
\hline 1999 & 25,0 & 33,3 & 41,7 & 7,8 & 20,3 & 71,9 \\
\hline 2000 & 0,0 & 50,0 & 50,0 & 4,6 & 18,5 & 76,9 \\
\hline 2001 & 7,7 & 15,4 & 76,9 & 7,0 & 13,9 & 79,1 \\
\hline 2002 & 7,7 & 23,1 & 69,2 & 5,8 & 19,8 & 74,4 \\
\hline 2003 & 8,3 & 25,0 & 66,7 & 4,0 & 19,0 & 77,0 \\
\hline 2004 & 13,3 & 6,7 & 80,0 & 1,0 & 17,0 & 82,0 \\
\hline 2005 & 14,2 & 42,9 & 42,9 & 2,4 & 13,4 & 84,2 \\
\hline 2006 & 33,3 & 25,0 & 41,7 & 4,8 & 8,4 & 86,8 \\
\hline
\end{tabular}




\section{REFERÊNCIAS}

Revista Brasileira de Fruticultura, Cruz das Almas, v.18, n.1, n.2, 1996; v.19, n.1, n.3, 1997; v.20, n.2, n.3, 1998; v.21, n.2, n.3, 1999; v.22, n.1, n.2, 2000; v.23, n.2, n.3, 2001; v.24, n.1, n.3, 2002; v.25, n.2, n.3, 2003; v.26, n.1, n.2, 2004; v.27, n.2, n.3, 2005; v.28, n.1, n.2, 2006.

Revista Fruits, Les Ulis, v.51, n.4, n.6, 1996; v.52, n.1, n.3, 1997; v.53, n.1, n.5, 1998; v.54, n.2, n.5, 1999; v.55, n.5, n.8,
2000; v.56, n.1, n.5, 2001; v.57, n.3, n.5-6, 2002; v.58, n.1, n.3, 2003; v.59, n.2, n.5, 2004; v.60, n.2, n.3, 2005; v.61, n.1, n.2, 2006.

RIPOLL, R.M. et al. Uso actual de la estadística en investigación biomédica: una comparación entre revistas de medicina general. Medicina Clínica, v.106, n.12, p.451456, 1996. 